\title{
BAFF Index and CXCL13 levels in the cerebrospinal fluid associate respectively with intrathecal $\lg G$ synthesis and cortical atrophy in multiple sclerosis at clinical onset
}

M. Puthenparampil ${ }^{1 *}$, L. Federle ${ }^{1}$, S. Miante ${ }^{1}$, A. Zito ${ }^{1}$, E. Toffanin² ${ }^{2}$ S. Ruggero², M. Ermani ${ }^{2}$, S. Pravato ${ }^{2}$, D. Poggiali ${ }^{1}$, P. Perini ${ }^{1}$, F. Rinaldi $^{1}$ and P. Gallo ${ }^{1}$

\begin{abstract}
Background: B lymphocytes are thought to play a relevant role in multiple sclerosis (MS) pathology. The in vivo analysis of intrathecally produced B cell-related cytokines may help to clarify the mechanisms of B cell recruitment and immunoglobulin production within the central nervous system (CNS) in MS.

Methods: Paired cerebrospinal fluid (CSF) and serum specimens from 40 clinically isolated syndrome suggestive of MS or early-onset relapsing-remitting MS patients (CIS/eRRMS) and 17 healthy controls $(\mathrm{HC})$ were analyzed for the intrathecal synthesis of IgG (quantitative formulae and IgG oligoclonal bands, IgGOB), CXCL13, BAFF, and IL-21. 3D-FLAIR, 3D-DIR, and 3D-T1 MRI sequences were applied to evaluate white matter (WM) and gray matter (GM) lesions and global cortical thickness (gCTh).

Results: Compared to $\mathrm{HC}$, CIS/eRRMS having IgGOB (IgGOB+, 26 patients) had higher intrathecal IgG indexes $(p<0.01)$, lower values of BAFF Index $(11.9 \pm 6.1$ vs $17.5 \pm 5.2, p<0.01)$, and higher CSF CXCL13 levels (27.7 \pm 33.5 vs $0.9 \pm 1.5, p<0.005)$. In these patients, BAFF Index but not CSF CXCL13 levels inversely correlated with the intrathecal IgG synthesis ( $r>0.5$ and $p<0.05$ for all correlations). CSF leukocyte counts were significantly higher in lgGOB+ compared to IgGOB- $(p<0.05)$ and HC $(p<0.01)$, and correlated to CSF CXCL13 concentrations $(r 0.77, p<0.001)$.

The gCTh was significantly lower in patients with higher CSF CXCL13 levels ( $2.41 \pm 0.1$ vs $2.49 \pm 0.1 \mathrm{~mm}, p<0.05)$, while no difference in MRI parameters of WM and GM pathology was observed between lgGOB+ and lgGOB-.

Conclusions: The intrathecal IgG synthesis inversely correlated with BAFF Index and showed no correlation with CSF CXCL13. These findings seem to indicate that intrathecally synthesized IgG are produced by long-term PCs that have entered the CNS from the peripheral blood, rather than produced by PCs developed in the meningeal follicle-like structures (FLS). In this study, CXCL13 identifies a subgroup of MS patients characterized by higher leukocyte counts in the CSF and early evidence of cortical thinning, further suggesting a role for this chemokine as a possible marker of disease severity.
\end{abstract}

Keywords: Multiple sclerosis, BAFF, CXCL13, IL21, Cerebrospinal fluid, IgGOB, FLS, Intrathecal IgG synthesis

\footnotetext{
* Correspondence: marco.puthenparampil@studenti.unipd.it

'Multiple Sclerosis Centre, Department of Neurosciences DNS, University

Hospital-Medical School, via Giustiniani 5, 3518 Padova, Italy

Full list of author information is available at the end of the article
} 


\section{Background}

Several lines of evidence suggest that B cells play a central role in the pathogenesis and/or the clinical evolution of multiple sclerosis (MS). First, it is well known that the great majority of MS patients have quantitative (increased indexes) and/or qualitative (presence of IgG oligoclonal bands, IgGOB) immunoglobulin changes in the cerebrospinal fluid (CSF), indicating an intrathecal Ig production [1, 2]. Second, histological studies have disclosed the presence of complement deposition and plasma cells (PCs) in white matter (WM) lesions [3]. Third, B cell follicle-like structures (FLS) are observed in the meninges of patients with progressive MS [4] and correlate with the degree of meningeal inflammation and cortical demyelination, and with a higher rate of disability progression [5]. Finally, the treatment of MS patients with anti-CD20 monoclonal antibodies determines a significant reduction in the number of both active brain lesions and in clinical relapses [6-9].

The role of $\mathrm{B}$ cells in MS pathology may be multifaceted [7]. Beside autoantibody production [10, 11], B cells may present central nervous system (CNS) antigens with co-stimulatory signals leading to activation and proliferation of effector $\mathrm{T}$ cells, may produce proinflammatory cytokines (e.g. IL-6, IL-12, and TNF), or may regulate the immune response by providing IL-10, a cytokine that suppresses the $\mathrm{T}$ cell functions either directly or indirectly [12] (i.e., differentiation of $\mathrm{T}$ regulatory cells and the suppression of dendritic cell function [7, 10-12]).

The presence of meningeal follicle-like structures (FLS) has stimulated the hypothesis of a "germinal center reaction" within the CNS of MS. Briefly, naive B cells recruited in the CNS (throughout CXCL13 signal) may locally meet and present CNS antigens to T helper lymphocytes. Both $\mathrm{B}$ and $\mathrm{T}$ cells actively collaborate (respectively as centroblast and follicular $\mathrm{T}$ helper, that produce IL-21, the "signature" of an ongoing follicular reaction) to establish a germinal center reaction that leads B cell maturation to IgG-secreting PCs. PCs will survive within the CNS thanks to intrathecally produced B cell survival factors, such as B cell-activating factor (BAFF), as suggested by a recent observation [13]. On the other hand, we cannot exclude that FLS formation may be a mere epiphenomenon of MS, expression of a chronic meningeal inflammation, not directly involved in intrathecal Ig synthesis.

We studied serum and CSF levels of soluble factors involved in naïve $B$ cell recruitment (CXCL13), in follicular reaction (IL-21) and intrathecal B cell survival (BAFF) in MS patients at clinical onset and correlated their intrathecal synthesis with local Ig production and with magnetic resonance imaging (MRI) parameters of white and gray matter damage. The aim of the study was to explore whether, at clinical onset, B cell-related cytokines could serve as immunological markers of MS severity/prognosis.

\section{Methods \\ Patients}

Forty consecutive patients with a diagnosis of clinically isolated syndrome suggestive of MS (CIS) or early-onset relapsing-remitting MS (eRRMS), whose diagnosis was achieved in agreement with the most recent diagnostic criteria [14], were enrolled in the study. Inclusion criteria were as follows: (i) age between 18 and 50 years, (ii) no medical history of steroid therapy in the previous 30 days, (iii) no medical history of immunomodulating or immunosuppressive therapy, and (iv) no gadoliniumenhancing lesion at the MRI performed at the study entry.

Seventeen healthy controls ( $\mathrm{HC}$ ) were also enrolled in the study. These subjects complained tension headache, subjective transient sensory symptoms, psychosomatic disorders, etc. that underwent lumbar puncture for diagnostic purposes having unexpected, subtle, but unspecific white matter lesions at MRI. Immunological and coagulation screenings, microbiological and standard CSF analysis were normal; also upper aortic artery and Transcranic ultrasound EcoColorDoppler and visual evoked potentials were normal.

No differences for age and gender were observed between eRRMS and HC (Table 1). The study was

Table 1 Standard serum and CSF parameters in HC and CIS/ eRRMS

\begin{tabular}{|c|c|c|}
\hline & $\mathrm{HC}$ & CIS/eRRMS \\
\hline Gender (F/M) & $13 / 4$ & $26 / 14$ \\
\hline Age at LP (years) & $43.2 \pm 9.2$ & $37.8 \pm 10.0$ \\
\hline Disease duration (years) & n.a. & $0.5 \pm 0.9$ \\
\hline CSF [Alb] (mg/dL) & $17.0 \pm 5.1$ & $22.5 \pm 9.8$ \\
\hline Serum [Alb] (mg/dL) & $4370.6 \pm 379.0$ & $4260.0 \pm 333.6$ \\
\hline $\mathrm{Q}_{\mathrm{Alb}}\left(10^{-3}\right)$ & $3.9 \pm 1.2$ & $5.3 \pm 2.3^{*}$ \\
\hline CSF $[\mathrm{lgG}](\mathrm{mg} / \mathrm{dL})$ & $2.2 \pm 0.9$ & $3.7 \pm 1.8^{* * *}$ \\
\hline Serum [lgG] (mg/dL) & $1141.2 \pm 215.2$ & $1042.0 \pm 197.7$ \\
\hline $\mathrm{Q}_{\lg G}\left(10^{-3}\right)$ & $1.9 \pm 0.6$ & $3.6 \pm 1.7^{* * * *}$ \\
\hline IgG Index & $0.5 \pm 0.1$ & $0.7 \pm 0.3^{* * *}$ \\
\hline IgG Loc (mg/dL) & $0.1 \pm 0.3$ & $5.9 \pm 10.2^{*}$ \\
\hline $\lg \mid \mathrm{F}(\%)$ & $0 \pm 0$ & $11 \pm 17^{*}$ \\
\hline $\operatorname{lgGOB}(\%)$ & $0 \%$ & $65 \% * * * *$ \\
\hline Leukocyte counts $(/ \mu \mathrm{L})$ & $2.1 \pm 1.1$ & $6.9 \pm 7.7^{*}$ \\
\hline
\end{tabular}

Disease duration was defined as the time between clinical disease onset and lumbar puncture. For gander, IgIF\% and IgGOB chi-square test was applied. For all the other variables, a $t$ test was performed

$H C$ healthy controls, CIS/eRRMS CIS suggestive of MS or early RRMS, LP lumbar puncture, n.a. not applicable, Csf cerebrospinal fluid, $S$ serum, $\lg G O B \lg G$ oligoclonal bands

${ }^{*} p<0.05 ;{ }^{* *} p<0.01 ;{ }^{* * *} p<0.005 ;{ }^{* * * *} p<0.001$ 
approved by the local ethics committee and a written informed consent was obtained by all the participants.

\section{CSF and serum routine analysis}

CSF was collected by non-traumatic lumbar puncture between 8:00 and 9:00 am. Routine examination on paired CSF and serum specimens included cell count and differentiation, CSF/serum IgG ratio $\left(\mathrm{Q}_{\mathrm{IgG}}\right)$, CSF/ serum albumin ratio $\left(\mathrm{Q}_{\mathrm{Alb}}\right)$ to estimate the integrity of the blood-brain barrier (BBB), calculation of intrathecal IgG synthesis by means of quantitative formulae (IgG Index) [2] IgG Reiber's Hyperbolic Function for IgG intrathecal synthesis fraction (IgGIF) [1] and Local Production (IgGLoc) [1], and demonstration of IgGOB by isoelectric-focusing and specific IgG immunofixation. BBB damage was considered when $\mathrm{Q}_{\mathrm{Alb}}$ was higher than the normal value for patient's age (i.e., age/15+4). After cell centrifugation, both CSF and serum were stored at $-80{ }^{\circ} \mathrm{C}$ until cytokine analysis.

\section{Cytokine determination}

CSF and serum levels of BAFF (R\&D System, Cat. Numb. DBLYS0B), IL-21 (Bender MedSystems Cat. Numb. BMS2043TEN), and CXCL13 (R\&D System, Cat. Numb. DCX130) were measured by a highly sensitive immune-enzymatic assay ELISA according to the manufacturer's instruction. BAFF concentrations $(\mathrm{pg} / \mathrm{mL})$ were measured on 1:2 diluted sera and on native CSF. IL-21 and CXCL13 concentrations $(\mathrm{pg} / \mathrm{mL})$ were measured on native sera and CSF. Cytokine ratio (CSF/ serum cytokine: $\mathrm{Q}_{\mathrm{BAFF}}, \mathrm{Q}_{\mathrm{CXCL13}}$, and $\mathrm{Q}_{\mathrm{IL}-21}$ ) and Index (CSF/serum cytokine/CSF/serum albumin: BAFF Index, CXCL13 Index, IL21 Index) were calculated.

\section{MRI protocol}

Images were acquired using a 3T scanner (Ingenia, Philips Medical Systems, Best, The Netherlands) with $33 \mathrm{mT} / \mathrm{m}$ power gradient and a 32-channel head coil. No major hardware upgrades occurred during the study, and bimonthly quality-assurance sessions assured measurement stability. The following images were acquired for each subject: (a) three-dimensional (3D) turbo field echo (TFE, 3D-T1): repetition time (RT) $7.8 \mathrm{~ms}$; echo time (ET) $3.6 \mathrm{~ms} ; 180$ contiguous axial slices with the off-center positioned on zero with thickness of $1.0 \mathrm{~mm}$; flip angle $=8^{\circ} ;$ matrix size $=220 \times 220 ; \quad$ FOV $=220 \times$ $220 \times 180 \mathrm{~mm}^{3}$. This sequence was acquired before and after gadolinium administration. (b) 3D-fluid attenuated inversion recovery (FLAIR): RT 4800 ms; ET $310 \mathrm{~ms}$; inversion time (IT) $1650 \mathrm{~ms} ; 365$ contiguous axial slices with thickness of $1.0 \mathrm{~mm}$; matrix size $256 \times 256$; and FOV $=256 \times 256 \times 182 \mathrm{~mm} 3$; (c) 3D-double inversion recovery (DIR): RT $13,000 \mathrm{~ms}$, ET $10 \mathrm{~ms}$, IT $3400 / 325 \mathrm{~ms}$; 40 contiguous axial slices, resolution $1 \times 1 \times 3 \mathrm{~mm}$, FOV
$230 \times 200 \mathrm{~mm}$, time $3.5 \mathrm{~min}$. Two experienced observers, blinded to the patient's identity, assessed all images. The global cortical thickness (gCTh) was analyzed by means of Freesurfer on 3D-T1 sequences. WM lesions were identified on FLAIR sequences, while cortical lesions (CL) were identified on DIR scans by two blinded evaluators (PD, PS) using published consensus recommendations.

\section{Statistical analysis}

For normally distributed variables, the $T$ test (when two groups were compared) or the ANOVA (for more than two group comparisons) were performed applying the Bonferroni correction. For ordinal categorical variables, the Mann-Whitney $U$ test was performed, while for no ordinal variables, Pearson's chi-square test was used. Linear correlation between variables was tested using Pearson's single or multiple linear model when all variables were normally distributed. The significance level was set at $p<0.05$.

\section{Results}

Intrathecal IgG synthesis in HC and CIS/eRRMS

Table 1 summarizes routine serum and CSF findings in $\mathrm{HC}$ and CIS/eRRMS. As expected, all the indexes of intrathecal IgG synthesis (IgG CSF, $Q_{\mathrm{IgG}}$, IgG Index, IgGLoc, and IgGIF) were significantly higher in CIS/ eRRMS compared to $\mathrm{HC}$. Higher $\mathrm{Q}_{\mathrm{Alb}}$ values were observed in CIS/eRRMS $\left(5.3 \pm 2.3 \times 10^{-3}\right)$ than in $\mathrm{HC}(3.9$ $\pm 1.2 \times 10^{-3}, p<0.05$ ), but only six CIS/eRRMS patients (15 vs $0 \%$ in $\mathrm{HC}, p=0.2$ ) had BBB damage.

\section{Intrathecal cytokine synthesis in HC and CIS/eRRMS CXCL13}

In $\mathrm{HC}, \mathrm{CXCL13}$ was found in all sera and, at very low levels $(0.9 \pm 1.5 \mathrm{pg} / \mathrm{mL})$, in $8 / 17$ (47\%) CSF. In these subjects, no correlation was observed between $\mathrm{Q}_{\text {CXCL13 }}$ and $\mathrm{Q}_{\text {Alb }}(r$ 0.2, $p=0.4)$, even when only CXCL13-positive CSF were included in the analysis $(r 0.7, p=0.1)$. Since these data indicated that CXCL13 was intrathecally produced, rather than derived from passive filtration from blood, only the CSF concentrations of this cytokine were considered in further analyses. CXCL13 was detected more frequently $(75 \%, p<0.05)$ and at higher concentration $(19.7 \pm 29.3 \mathrm{pg} / \mathrm{mL}, p<0.05)$ in the CSF of CIS/ eRRMS than HC.

\section{IL-21}

In $\mathrm{HC}$, IL-21 was detectable in all CSF (28.1 \pm $29.1 \mathrm{pg} / \mathrm{mL}$ ) but only in one serum, thus suggesting that CSF IL-21 was intrathecally produced. No difference in CSF IL-21 levels was observed between HC (28.1 \pm $29.1 \mathrm{pg} / \mathrm{mL})$ and CIS/eRRMS $(20.7 \pm 22.8 \mathrm{pg} / \mathrm{mL}, p=0.3)$. 


\section{BAFF}

BAFF was detected in all the sera and CSF of $\mathrm{HC}$ and CIS/eRRMS. In $\mathrm{HC} \mathrm{Q}_{\mathrm{BAFF}}$ mildly correlated to

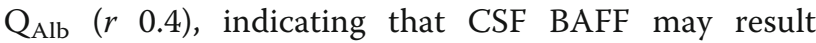
from both choroid plexus filtration and active production within the CNS. The BAFF Index was lower in CIS/eRRMS $(12.4 \pm 5.5 \mathrm{pg} / \mathrm{mL})$ than in $\mathrm{HC}(17.5 \pm$ $5.2 \mathrm{pg} / \mathrm{mL}, p<0.005)$.

\section{IgGOB-based stratification of CIS/eRRMS}

Based on the presence of IgGOB in the CSF, CIS/eRRMS patients were divided in IgGOB positive (IgGOB+, 26 patients) and in IgGOB negative (IgGOB-, 14 patients) (Table 2). The analysis based on this stratification disclosed that all the differences previously found between $\mathrm{HC}$ and CIS/eRRMS were confirmed for IgGOB+ patients, but not for IgGOB- patients (Fig. 1a). Furthermore, serum IgG concentrations in IgGOB- were lower than in $\mathrm{HC}(p<0.01)$ and IgGOB $+(p<0.05)$.

CSF CXCL13 concentrations were higher in IgGOB+ compared to both IgGOB- $(p<0.05)$ and HC $(p<0.005)$ (Fig. 1b). A higher number of IgGOB+ patients had detectable CXCL13 in the CSF (77\%) compared to IgGOB(50\%), but the difference was not significant $(p=0.2)$.

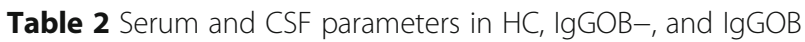
+ patients

\begin{tabular}{|c|c|c|c|}
\hline & $\mathrm{HC}$ & $\operatorname{lgGOB}-$ & $\operatorname{lgGOB+}$ \\
\hline Gender (F/M) & $13 / 4$ & $9 / 5$ & $17 / 9$ \\
\hline Age at LP (years) & $43.2 \pm 9.2$ & $37.2 \pm 10.7$ & $38.2 \pm 9.7$ \\
\hline $\begin{array}{l}\text { Disease duration } \\
\text { (years) }\end{array}$ & n.a. & $0.3 \pm 0.6$ & $0.6 \pm 1.1$ \\
\hline Csf-[Alb] (mg/dL) & $17.0 \pm 5.1$ & $21.2 \pm 8.7$ & $23.1 \pm 10.4$ \\
\hline $\mathrm{S}-[\mathrm{Alb}](\mathrm{mg} / \mathrm{dL})$ & $4370.6 \pm 379.0$ & $4225.0 \pm 297.7$ & $4278.8 \pm 355.6$ \\
\hline$Q_{A l b}\left(10^{-3}\right)$ & $3.9 \pm 1.2$ & $5.0 \pm 2.0$ & $5.4 \pm 2.4^{*}$ \\
\hline Csf-[lgG] (mg/dL) & $2.2 \pm 0.9$ & $2.4 \pm 0.8$ & $4.5 \pm 1.8^{* * * * 0000}$ \\
\hline $\mathrm{S}-[\mathrm{lgG}](\mathrm{mg} / \mathrm{dL})$ & $1141.2 \pm 215.2$ & $944.6 \pm 124.2^{* *}$ & $1094.3 \pm 211.6^{\circ}$ \\
\hline$Q_{\operatorname{lgG}}\left(10^{-3}\right)$ & $1.9 \pm 0.6$ & $2.6 \pm 1.0$ & $4.2 \pm 1.8^{* * * * \circ}$ \\
\hline IgG Index & $0.5 \pm 0.1$ & $0.5 \pm 0.1$ & $0.8 \pm 0.3^{* * * * 000}$ \\
\hline $\operatorname{lgG} \operatorname{Loc}(\mathrm{mg} / \mathrm{dL})$ & $0.1 \pm 0.3$ & $0.2 \pm 0.6$ & $9.0 \pm 11.5^{* * \circ}$ \\
\hline $\lg \mid \mathrm{F}(\%)$ & $0 \pm 0$ & $1.1 \pm 2.7$ & $16.4 \pm 18.5^{* * * \circ \circ}$ \\
\hline 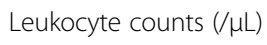 & $2.1 \pm 1.1$ & $2.9 \pm 2.4$ & $9.0 \pm 8.6^{* * * \circ}$ \\
\hline Csf -[BAFF] (pg/mL) & $67.3 \pm 21.6$ & $69.9 \pm 27.3$ & $50.7 \pm 20.5^{*} \circ$ \\
\hline BAFF Index & $17.5 \pm 5.2$ & $13.2 \pm 5.1$ & $11.9 \pm 6.1^{* *}$ \\
\hline Csf-[CXCL13] (pg/mL) & $0.9 \pm 1.5$ & $5.0 \pm 8.2$ & $27.7 \pm 33.5^{* * * \circ}$ \\
\hline Csf-[lL21] (pg/mL) & $28.1 \pm 29.1$ & $21.6 \pm 25.3$ & $20.2 \pm 21.9$ \\
\hline
\end{tabular}

$\mathrm{lgGOB}+$ patients differed from both BOlgG- and $\mathrm{HC}$ for intrathecal IgG synthesis parameters, CSF CXCL13 concentrations, and BAFF Index values. For gender, IgIF\%, and IgGOB, chi square test was applied. For all the other variables, a $t$ test was performed. Abbreviations as in Table 1

$P$ values compared to HC: ${ }^{*} p<0.5 ;{ }^{* *} p<0.01 ;{ }^{* * *} p<0.005 ;{ }^{* * * *} p<0.001$;

$p$ values compared to IgGOB-: ${ }^{\circ} p<0.5 ;{ }^{\circ 0} p<0.01 ;{ }^{\circ 00} p<0.005 ;{ }^{\circ 000} p<0.001$
No difference in CSF IL-21 levels was observed between $\mathrm{HC}$, IgGOB-, and IgGOB+ (Fig. 1c).

As expected on the base of previous results, CSF BAFF levels and Index were significantly lower in IgGOB $+(50.7 \pm 20.5 \mathrm{pg} / \mathrm{ml}, p<0.05$, and $11.9 \pm 6.1$, both $p<0.005$, respectively) but not in IgGOB- (69.9 $\pm 27.3 \mathrm{pg} / \mathrm{ml}$ and $13.2 \pm 5.1$, respectively, $p=0.8$ and $p=0.1$ ) compared to HC (Fig. 1d).

\section{Correlation analysis}

For the correlation analysis, only quantitative continuous variables, namely IgG Index, IgGLoc, and IgGIF, were included as dependent variables. Table 3 shows the correlation between the parameters of intrathecal IgG synthesis and BAFF Index or CSF CXCL13 concentrations in IgGOB+. CSF CXCL13 concentrations correlated with all the parameters of intrathecal $\mathrm{IgG}$ synthesis (Fig. 2a-c), while BAFF Index mildly correlated only with IgGLoc values (Fig. 3a-c). In order to avoid the influence of null values on the direct correlation and considering that IgGOB + presented null values for CSF CXCL13, we focused on patients with quantitatively increased intrathecal IgG synthesis. In 15 IgGOB+ patients having IgG Index, IgGLoc, and IgGIF values above the normal range of reference, only BAFF Index correlated to all these parameters (Fig. 3a'-c'), while CXCL13 concentrations correlated only with IgGLoc (Fig. 2a').

Finally, BAFF Index did not correlate to CSF CXCL13 concentrations in $\mathrm{HC}(r-0.36, p=0.2)$, MS patients $(r-0.29, p=0.1)$, IgGOB $-(r-0.05, p=0.9)$, and IgGOB+ $(r-0.31, p=0.1)$.

\section{CSF leukocyte counts in CIS/eRRMS and in IgGOB+ and IgGOB-}

CSF leukocyte number was significantly higher in CIS/eRRMS $(6.9 \pm 7.7 / \mu \mathrm{L})$ compared to $\mathrm{HC}(2.1 \pm$ $1.1 \mu \mathrm{L}, p<0.05)$. CSF CXCL13 concentrations, but not BAFF Index, significantly correlated to leukocyte counts ( $r$ 0.77, $p<0.001$, Table 4 ).

BOIgG+ had higher CSF leukocyte number (9.0 \pm $8.6 / \mu \mathrm{L})$ than IgGOB- $(2.9 \pm 2.4 / \mu \mathrm{L}, p<0.05)$ and $\mathrm{HC}$ $(p<0.01)$. In IgGOB + , CSF CXCL13 concentrations, but not BAFF Index values, significantly correlated to leukocyte number $(r$ 0.77, $p<0.001$, Table 4$)$, while no correlation between leukocyte number and all the other CSF parameters was observed in BOIgG-.

\section{MRI parameters in CIS/eRRMS}

No difference in gCTh was observed between $\mathrm{HC}$ and CIS/eRRMS. However, a higher variability in gCTh was found in CIS/eRRMS (range 2.25-2.67 mm) compared to $\mathrm{HC}(2.34-2.58 \mathrm{~mm})$. In order to find associations 


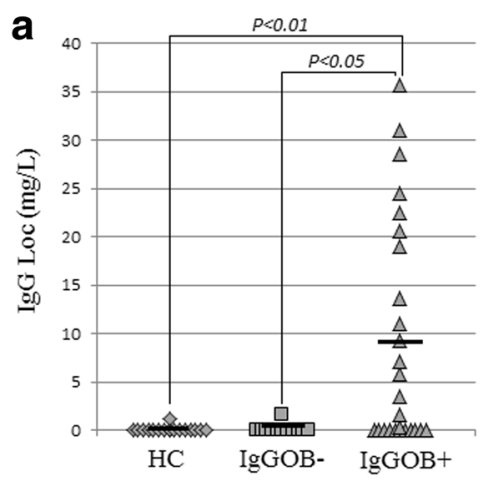

C

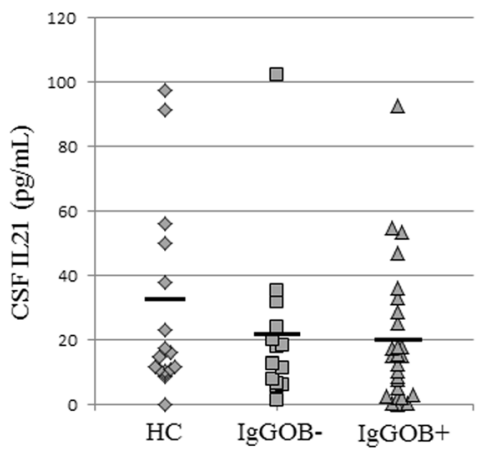

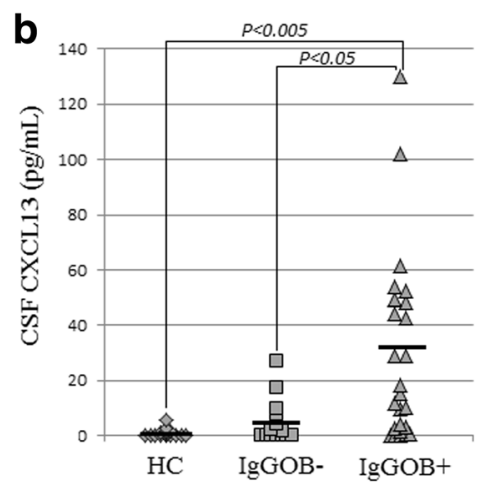

d

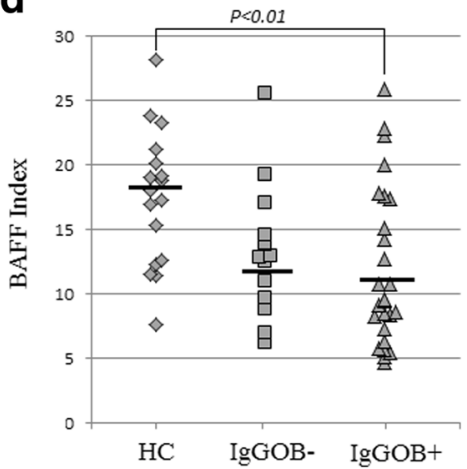

Fig. $1 \mathrm{lgG}$ and cytokine concentrations in the CSF. IgG Loc expressed in mg/dL (a), CSF CXCL13 and IL-21 concentrations (pg/mL; b, c), and BAFF Index values (d) in $\mathrm{HC}$ and $\mathrm{CIS} / \mathrm{eRRMS}$ with (IgGOB+) or without (IgGOB-) intrathecal synthesis of IgG oligoclonal bands. IgGOB+ patients had increased CXCL13, normal IL-21, and decreased BAFF Index compared to HC and IgGOB- patients. Only significant comparison is indicated. Abbreviations: HC healthy controls, CIS/eRRMS CIS suggestive of MS or early RRMS, LP lumbar puncture, n.a. not applicable, CSF cerebrospinal fluid, $S$ serum. IgGOB+: patients with IgG oligoclonal bands in the CSF; IgGOB-: patients without lgG oligoclonal bands in the CSF

between CSF (IgGOB detection, BAFF Index, CSF CXCL13, IgG Index, IgGLoc, IgG IF) and MRI parameters, we performed a subgroup analysis.

On the base CXCL13 concentrations in the CSF of $\mathrm{HC}$, we found a cutoff value of $6.8 \mathrm{pg} / \mathrm{mL}(\mu+4 \delta)$ that allowed the identification of two groups of CIS/eRRMS patients having CSF CXCL13 values below (CXCL13-,

Table 3 Correlation between intrathecal lgG synthesis parameters and BAFF Index or CXCL13

\begin{tabular}{lll}
\hline (A) All patients & BAFF Index \\
IgG Loc (mg/dL) & $-0.41^{*}$ & $0.59^{* * *}$ \\
$\lg \mid \mathrm{F}(\%)$ & -0.36 & $0.45^{*}$ \\
$\lg$ Index & -0.38 & $0.48^{*}$ \\
(B) Only IS+ & BAFF Index & LCS-CXCL13 \\
IgG Loc (mg/dL) & $-0.56^{*}$ & $0.57^{*}$ \\
IgIF (\%) & $-0.52^{*}$ & 0.35 \\
IgG Index & $-0.64^{*}$ & 0.43 \\
\hline
\end{tabular}

The correlation was calculating on overall population of BOlgG+ patients (A) or including only patients with quantitatively increased intrathecal lgG synthesis (IS+, B). $R$ values derived from Pearson's single linear model are represented. Other abbreviations as in Table 2

${ }^{*} p<0.5 ;{ }^{* *} p<0.01 ;{ }^{* * *} p<0.005$
20 patients) or above (CXCL13+, 20 patients) this limit. All HC had CXCL13 values below this limit.

BAFF Index was not applicable in this analysis, since its range did not differ between the two groups and therefore a cut-off value was meaningless.

In as much as the three quantitative indexes of intrathecal IgG synthesis gave highly concordant values, only IgG Loc was considered for further analysis (values $\leq 0.0=$ IgGLoc - ; values $>0.0=$ IgGLoc + ).

Interestingly, no difference in any MRI parameter was observed between IgGOB+ and IgGOB- or between IgGLoc- and IgGLoc+. CXCL13+ patients had a significant thinning of gCTh $(2.42 \pm 0.09 \mathrm{~mm})$ compared to CXCL13- $(2.48 \pm 0.10 \mathrm{~mm}, p<0.05$; Fig. 4). The results did not change when a different cut-off value $(\mu+3 \delta$, i.e., $5.2 \mathrm{pg} / \mathrm{mL}$ ) was applied.

No association was found between any CSF parameters and WM or GM lesion number and volume.

\section{Discussion}

Intrathecally produced IgGOB can be demonstrated in the great majority of MS patients at clinical onset, persist in the CSF throughout the patient's life, and constitute the major evidence of B lymphocytes and PC involvement in MS 

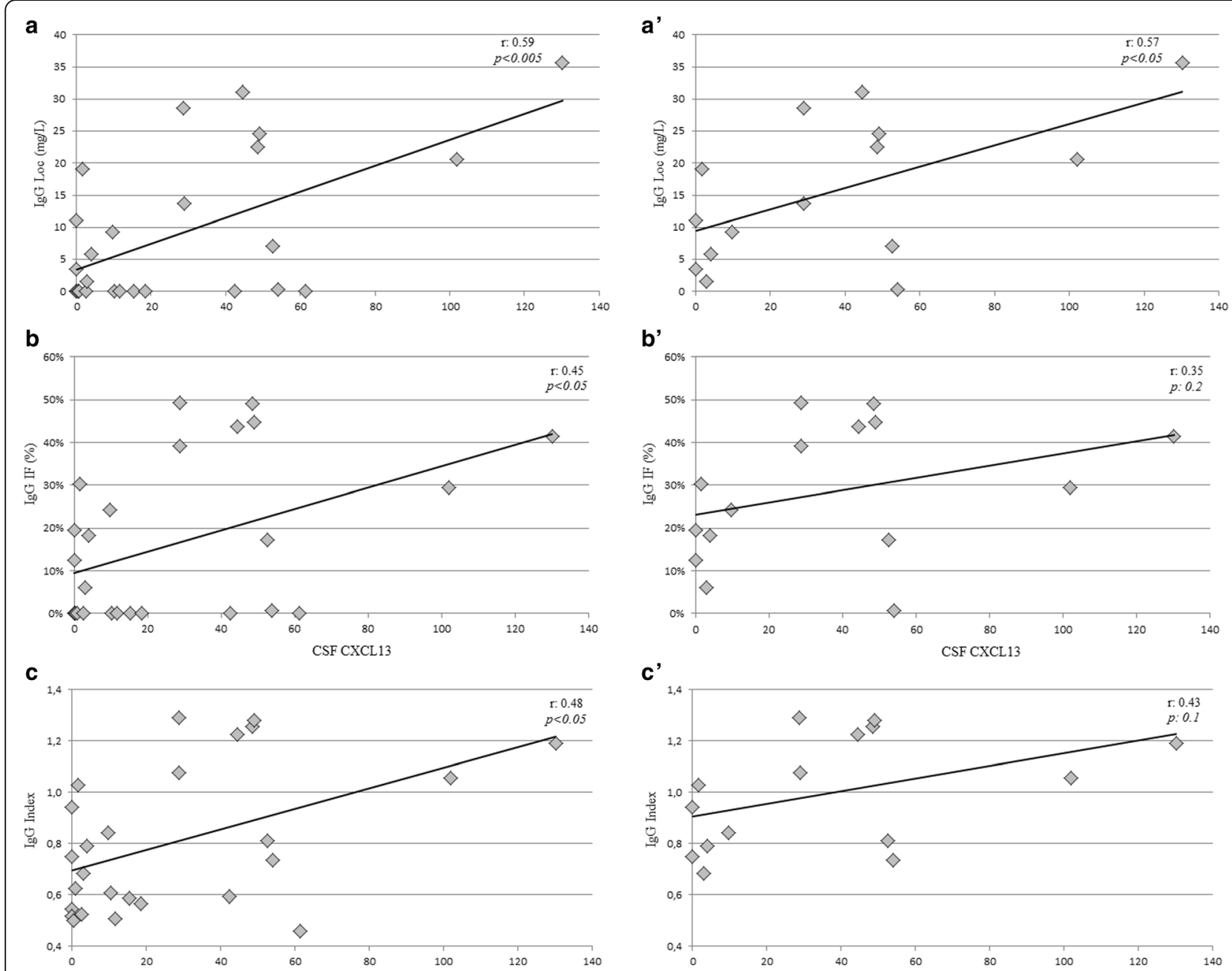

Fig. 2 Correlations between CSF CXCL13 and intrathecal lgG synthesis. A mild correlation was observed between CSFCXCL13 and intrathecal IgG synthesis when all the patients were included in the analysis $(\mathbf{a}-\mathbf{c})$. When the null values for intrathecal IgG synthesis were excluded, the correlation was weak $\left(\mathbf{a}^{\prime}\right)$ or lost $\left(\mathbf{b}^{\prime}, \mathbf{c}^{\prime}\right)$

pathology [3]. The origin of long-term secreting PCs, likely the source of intrathecally synthesized IgG, has not been established yet. Moreover, the progressive colonization of MS meninges by FLS suggests that local B cell maturation to PCs may support the intrathecal synthesis of IgM and IgG [5]. However, since FLS accumulation seems to parallel disease progression (i.e., they were demonstrated in high percentage in progressive MS cases, rarely in RRMS and not at clinical onset), we would expect to observe qualitative modifications of the IgGOB patterns over the course of the disease. On the contrary, longitudinal studies demonstrated that IgGOB are almost qualitatively stable [15-19].

$B$ cells have several other functions than producing antibodies [12]. They may act as efficient antigenpresenting or cytokine-secreting cells, thus enhancing $\mathrm{T}$ cell response. Therefore, the pathogenic role of $\mathrm{B}$ cells in the FLS may be multifaceted. FLS have been observed in association with cortical inflammation, gray matter demyelination, and various degrees of microglia activation [4]. Moreover, meningeal and cortical inflammation were found to correlate with some clinical parameters, such as median age at disease onset, time to disease progression, time to wheelchair dependence, and age at death [4]. Thus, the analysis of intrathecally synthesized B cell-related cytokines/chemokines may give the opportunity to define the timing of $\mathrm{B}$ cell recruitment and FLS formation in the CNS and to explore the role of FLS in the pathogenesis and clinical progression of MS.

In a previous study [13], we observed that BAFF Index correlated to quantitative parameters of intrathecal IgG synthesis in MS at clinical onset and suggested a BAFFdependent PCs survival within the CNS. In this study, we analyzed the intrathecal production of cytokines involved in B cell recruitment and follicular maturation in order to explore the origin of intracerebral PCs. 

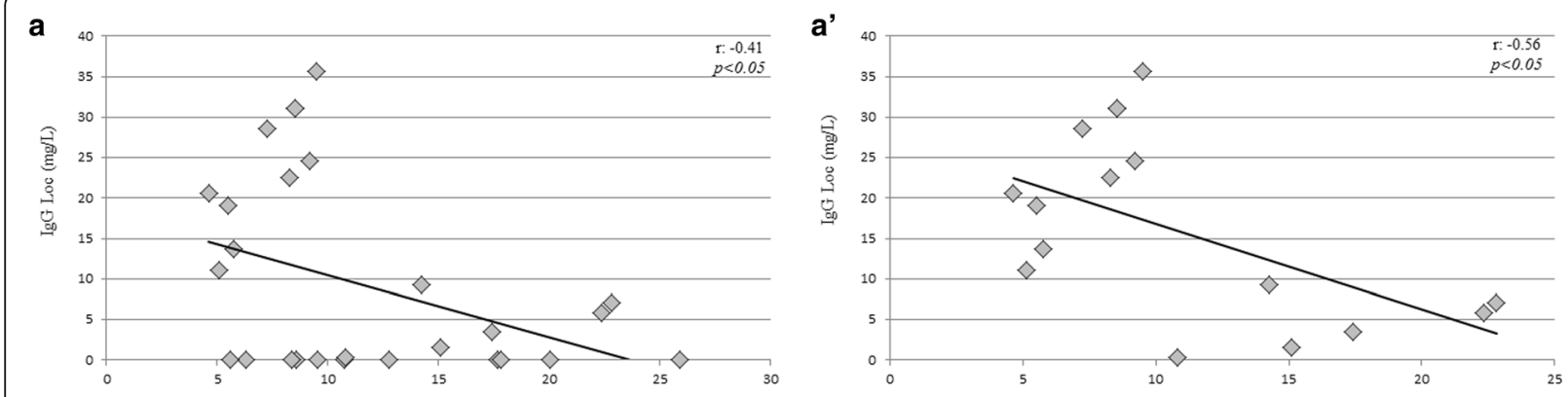

b

b'
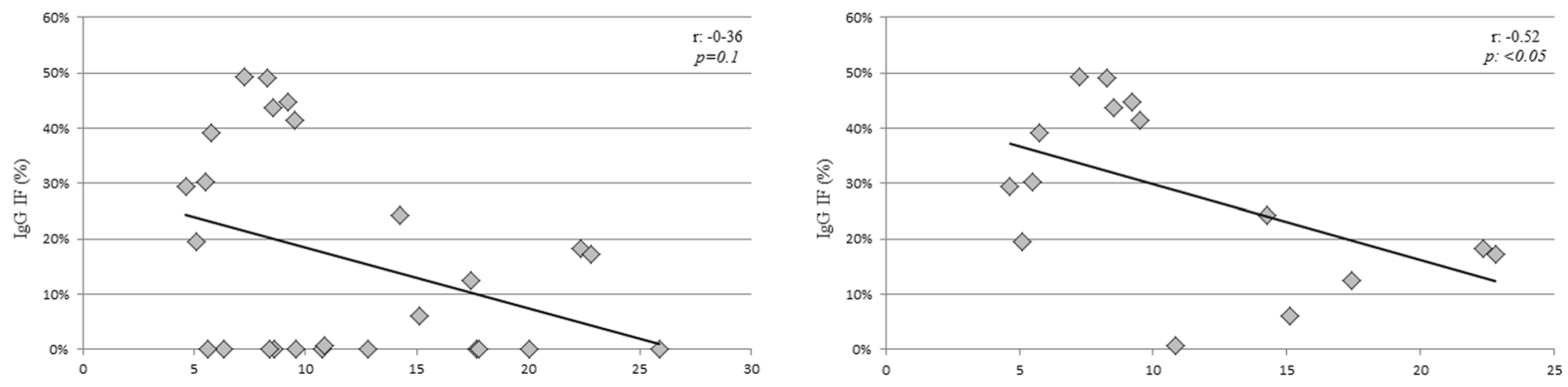

$c^{\prime}$
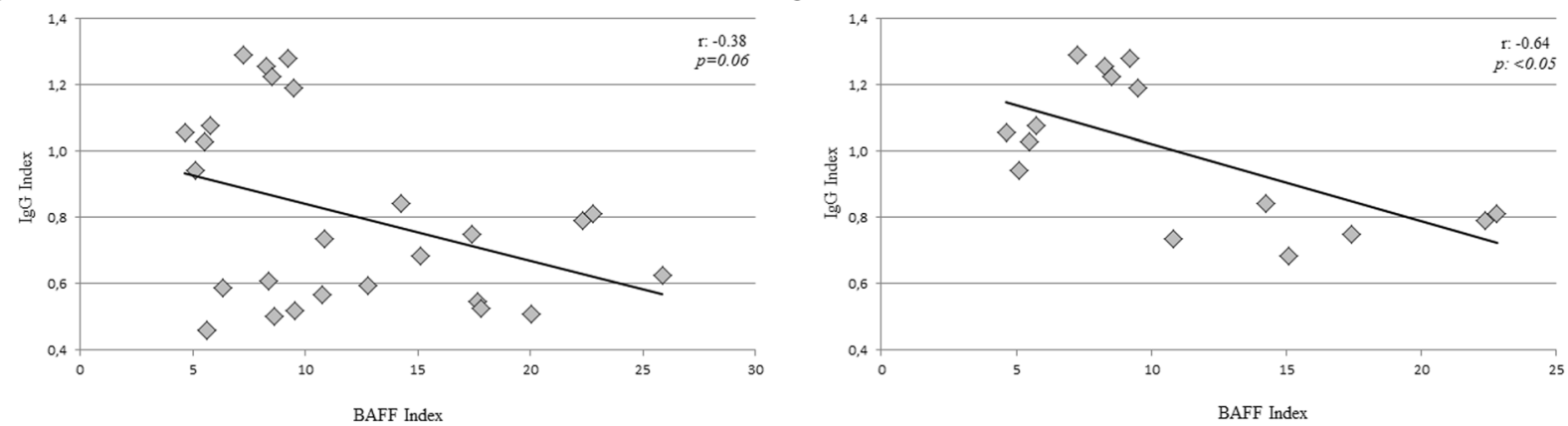

Fig. 3 Correlations between BAFF Index and intrathecal IgG synthesis. A mild correlation between BAFF Index and intrathecal IgG synthesis was observed when all the patients were considered $(\mathbf{a}-\mathbf{c})$. The correlation was strong when the null values of intrathecal lgG synthesis were excluded $\left(\mathbf{a}^{\prime}-\mathbf{c}^{\prime}\right)$

Table 4 Leukocyte counts in CIS/eRRMS correlates to CSF-CXCL13

\begin{tabular}{llll}
\hline & $r$ & $r^{2}$ & $p$ value \\
\hline CIS/eRRMS & & & \\
CSF-[CXCL13] (pg/mL) & 0.79 & $63.2 \%$ & $<0.001$ \\
BAFF Index & -0.21 & $4.4 \%$ & 0.2 \\
IgGOB+ & & & \\
CSF-[CXCL13] (pg/mL) & 0.77 & $59.3 \%$ & $<0.001$ \\
BAFF Index & -0.22 & $4.7 \%$ & 0.3 \\
IgGOB- & & & \\
CSf-[CXCL13] (pg/mL) & 0.34 & $11.6 \%$ & 0.3 \\
BAFF Index & 0.22 & $4.8 \%$ & 0.5 \\
\hline
\end{tabular}

Subgroup analysis revealed that this correlation was confirmed only in lgGOB+ patients. Abbreviations as in Table 2. $R$ values derived from Pearson's single linear model are represented
CXCL13, a chemokine that is not physiologically produced within the CNS, was increased in the CSF of CIS/ eRRMS, especially in IgGOB+ patients. This finding suggests that CXCL13, produced by activated microgial cells, becomes detectable since the early disease phases, and is responsible for the recruitment of naïve $B$ cells within the CNS. On the other hand, BAFF, constitutively produced in the CNS by astrocytes, is absorbed by local antibody-producing PCs, thus explaining the decreased levels of this cytokine in early MS stages. The previously described increased BAFF levels in the CSF of progressive MS [20] might found an explanation in the more diffuse astroglial proliferation and in the decreased burden of inflammation that characterize the more advanced phase of MS. However, since CSF CXCL13 was detected, although rarely, in patients with no evidence of intrathecal IgG synthesis, is not associated with 


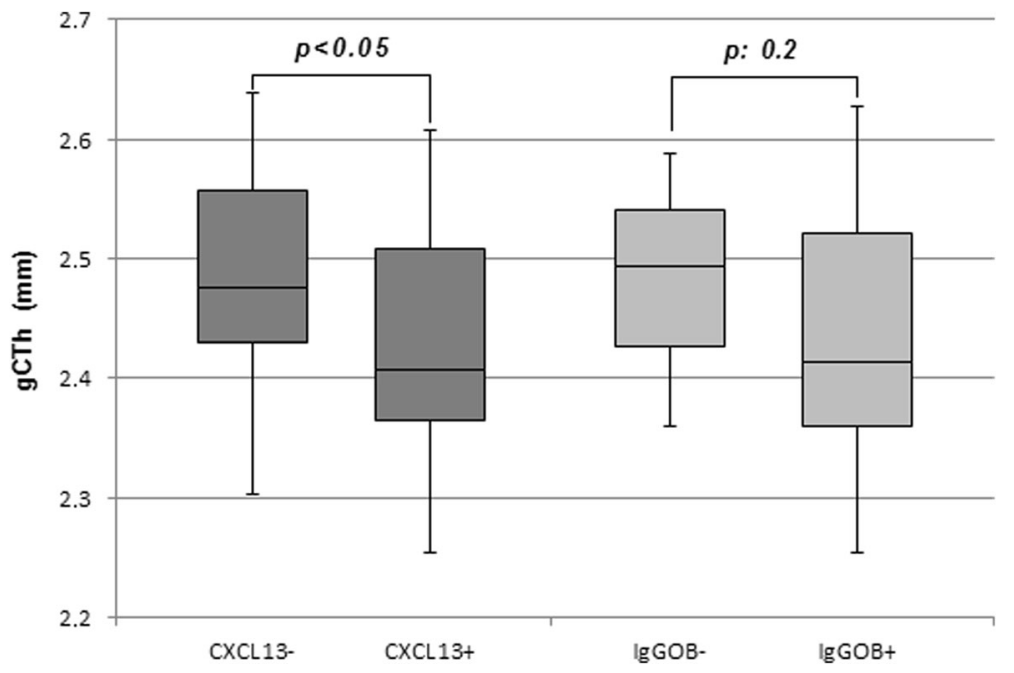

Fig. 4 Global cortical thickness is reduced in patients with higher CSF CXCL13. MS patients with higher CSF CXCL13 (CXCL13+) concentrations presented a significant cortical thinning compared to patients with lower values (CXCL13-)

BAFF Index and correlates only mildly with IgGLoc, we may argue that the majority of naïve $B$ cells recruited in the CNS do not undergo the process of maturation to PCs. Taken all together, these findings do not speak in favor of a germinal center reaction within FLS, at least in early MS phases, and are in line with the qualitative stability of IgGOB patterns during the course of MS.

An interesting finding of our study was the association of CSF CXCL13, but not BAFF Index, with cortical thinning since early MS phases. This observation seems to link a soluble factor that plays a pivotal role in the B cell recruitment in the CNS, to a MRI parameter of neurodegeneration (gray matter atrophy), that is correlated to disability progression in MS.

Our findings are in line with the previous reports on the association of CXCL13 to leukocyte CSF counts [20], higher risk of relapse, and CIS conversion to MS [21], as well as with higher disease activity in the WM $[22,23]$, further suggesting a possible role of CXCL13 as a soluble marker of disease activity or severity in MS.

Other cytokines were found to increase in MS CSF [24]. Among these, the macrophage/microglia-derived interleukin-1 beta (IL-1 $\beta$ ) was also found to correlate with cortical thinning [25]. The finding that both IL-1 $\beta$ and CXCL13 can be found in the CSF of very early MS and both factors associate with cortical thinning further suggests a pivotal role of microglia in MS pathology. Indeed, in vitro, CXCL13 is produced by monocytes, and at much higher levels by macrophages, and CXCL13 mRNA and protein expression is induced by TNF $\alpha$ and IL-1 $\beta$ [26]. All these data suggest that activated and proliferating microglia cells (a characteristic histological feature of cortical pathology) produce large amounts cytokines, such as IL-1 $\beta$ and CXCL13, that attract and maintain $\mathrm{T}$ and $\mathrm{B}$ cells in the inflamed meninges and in the cortex of MS.

\section{Conclusions}

Our findings indicate that intrathecally synthesized IgG are produced by PCs that enter the CNS from the peripheral blood at a given time and are locally retained as long-term PCs. A role for FLS as source of intrathecal IgG in early disease phases is not supported by our observations on CXCL13.

In our study, CXCL13 identifies a subgroup of MS patients characterized by the early evidence of cortical thinning, further suggesting a role for this chemokine as marker of disease severity.

\section{Abbreviations}

BAFF: B cell-activating factor; BBB: Blood brain barrier; CIS: Clinical isolated syndrome; CL: Cortical lesions; CNS: Central nervous system; CSF: Cerebrospinal fluid; DIR: Double inversion recovery; eRRMS: Early-onset relapsing-remitting multiple sclerosis; FLAIR: Fluid attenuated inversion recovery; HC: Healthy control; IgGIF: IgG intrathecal fraction; IgGLoc: IgG locally produced; IgGOB: IgG oligoclonal bands; MS: Multiple sclerosis; PCs: Plasma cells; $Q_{A \mathrm{Alb}}$ : CSF/serum

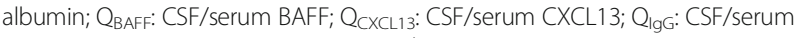
IgG ratio; $\mathrm{Q}_{\mathrm{LL}-21}$ : CSF/serum IL-21; WM: White matter

\section{Acknowledgements}

The authors would like to thank the patients and staff at the Multiple Sclerosis Centre of Padua, especially the nurses Elena Bortolami, Irene Boscariol, and Elisa Quaggia.

\section{Funding}

This research was supported by the grants from the Italian Minister of Public Health, Project GR-2010-2313255.

Availability of data and materials

Please contact the author for data requests.

\section{Authors' contributions}

PM, FL, and SM contributed to the concept and design of the study and performed clinical data acquisition, analysis, and interpretation and they 
performed a critical revision of the manuscript for important intellectual content. AZ and ME performed the data analysis and interpretation and they performed a critical revision of the manuscript for important intellectual content. ET and SR performed the CSF and serum analysis and they performed a critical revision of the manuscript for important intellectual content. SP and DP performed the acquisition, analysis, and interpretation of MRI data. RF, PP, and GP contributed to the concept of the study and performed a critical revision of the manuscript for important intellectual content. All authors read and approved the final manuscript.

\section{Competing interests}

Puthenparampil Marco received a travel grant from Novartis, Genzyme, Biogen Idec, Teva and Sanofi Aventis; he has been a consultant for Genzyme. Federle Lisa has received funding for travel from Novartis, Merck Serono, Biogen Idec, Sanofi-Aventis, Bayer Schering Pharma, Almirall, Genzyme, Teva and honoraria from MerkSerono, Teva and Almirall. Miante Silvia received a travel grant form Biogen Idec, Novartis, Sanofi Aventis and Teva. Zito Antonio, Toffanin Elisabetta, Ruggiero Susanna, Mario Ermani, and Stefano Pravato have nothing to disclose. Davide Poggiali received a travel grant from Biogen Idec, Novartis, Sanofi Aventis and Teva. Perini Paola has received funding for travel and speaker honoraria from Merck Serono, Biogen Idec, Sanofi-Aventis, and Bayer Schering Pharma and has been a consultant for Merck Serono, Biogen Idec and Teva; Midena Edoardo has nothing to disclose Rinaldi Francesca serves as an advisory board member of Biogen-Idec and has received funding for travel and speaker honoraria from Merck Serono, Biogen Idec, Sanofi-Aventis, Teva and Bayer Schering Pharma. Gallo Paolo has been a consultant for Bayer Schering, Biogen Idec, Genzyme, Merck Serono and Novartis, has received funding for travel and speaker honoraria from Merck-Serono, Biogen Idec, Sanofi-Aventis, Novartis Pharma and Bayer-Schering Pharma, Teva, has received research support from Bayer, Biogen Idec/Elan, MerkSerono, Genzyme and Teva, and has received research grant from the University of Padova, Veneto Region of Italy, the Italian Association for Multiple Sclerosis, the Italian Ministry of Public Health.

\section{Consent for publication}

Not applicable.

\section{Ethics approval and consent to participate}

The study was approved by the local ethics committee and a written informed consent was obtained by all the participants.

\section{Author details}

'Multiple Sclerosis Centre, Department of Neurosciences DNS, University Hospital-Medical School, via Giustiniani 5, 3518 Padova, Italy. ${ }^{2}$ Department of Neurosciences DNS, University Hospital-Medical School, via Giustiniani 5, 3518 Padova, Italy.

Received: 22 August 2016 Accepted: 28 December 2016 Published online: 17 January 2017

\section{References}

1. Reiber H, Peter JB. Cerebrospinal fluid analysis: disease-related data patterns and evaluation programs. J Neurol Sci. 2001;184:101-22.

2. Link $H$, Tibbling $G$. Principles of albumin and IgG analyses in neurological disorders. III. Evaluation of IgG synthesis within the central nervous system in multiple sclerosis. Scand J Clin Lab Invest. 1977;37:397-401.

3. Lucchinetti C, Bruck W, Parisi J, Scheithauer B, Rodriguez M, Lassmann H. Heterogeneity of multiple sclerosis lesions: implications for the pathogenesis of demyelination. Ann Neurol. 2000;47:707-17.

4. Howell OW, Reeves CA, Nicholas R, Carassiti D, Radotra B, Gentleman SM, et al. Meningeal inflammation is widespread and linked to cortical pathology in multiple sclerosis. Brain. 2011;134:2755-71.

5. Magliozzi R, Howell O, Vora A, Serafini B, Nicholas R, Puopolo M, et al. Meningeal B-cell follicles in secondary progressive multiple sclerosis associate with early onset of disease and severe cortical pathology. Brain. 2007;130:1089-104.

6. Hauser SL, Waubant E, Arnold DL, Vollmer T, Antel J, Fox RJ, et al. Bcell depletion with rituximab in relapsing-remitting multiple sclerosis. N Engl J Med. 2008;358:676-88.
7. Krumbholz M, Derfuss T, Hohlfeld R, Meinl E. B cells and antibodies in multiple sclerosis pathogenesis and therapy. Nat Rev Neurol. 2012;8:613-23.

8. He D, Guo R, Zhang F, Zhang C, Dong S, et al. Rituximab for relapsingremitting multiple sclerosis. Cochrane Database Syst. Rev. 2013.

9. Hauser SL, Comi GC, Hartung HP, Selmaj K, Traboulsee A, Bar-Or A, et al. Oral Presentation n. 190: efficacy and safety of ocrelizumab in relapsing multiple sclerosis - results of the interferon-beta-1a-controlled, doubleblind, phase III OPERA I and II studies. Mult Scler J. 2015;21:21.

10. Owens GP, Bennett JL, Lassmann H, O'Connor KC, Ritchie AM, Shearer A, et al. Antibodies produced by clonally expanded plasma cells in multiple sclerosis cerebrospinal fluid. Ann Neurol. 2009;65:639-49.

11. Obermeier B, Lovato L, Mentele R, Brück W, Forne I, Imhof A, et al. Related B cell clones that populate the CSF and CNS of patients with multiple sclerosis produce CSF immunoglobulin. J Neuroimmunol. 2011.

12. Thaunat $\mathrm{O}$, Morelon E, Defrance T. Am\&quot;B\&quot;valent: anti-CD20 antibodies unravel the dual role of B cells in immunopathogenesis. Blood. 2010;116:515-21.

13. Puthenparampil M, Miante S, Federle L, Zanetta C, Toffanin E, Ruggero S, et al. BAFF is decreased in the cerebrospinal fluid of multiple sclerosis at clinical onset. J Neuroimmunol. 2016;297:63-7.

14. Filippi M, Rocca MA, Ciccarelli O, De Stefano N, Evangelou N, Kappos L, et al. MRI criteria for the diagnosis of multiple sclerosis: MAGNIMS consensus guidelines. Lancet Neurol. 2016;15:292-303.

15. Petzold A, Abraira V, Alvarez-Cermeño JC, Arroyo R, Cámara C, Casanova B, et al. Intrathecal oligoclonal lgG synthesis in multiple sclerosis. J Neuroimmunol. 2013;262:1-10.

16. Link H, Huang Y-M. Oligoclonal bands in multiple sclerosis cerebrospinal fluid: an update on methodology and clinical usefulness. J Neuroimmunol. 2006;180:17-28.

17. Correale J, de los Milagros Bassani Molinas M. Oligoclonal bands and antibody responses in multiple sclerosis. J Neurol. 2002;249:375-89.

18. Confavreux C, Chapuis-Cellier C, Arnaud P, Robert O, Aimard G, Devic M. Oligoclonal \&quot;fingerprint\&quot; of CSF IgG in multiple sclerosis patients is not modified following intrathecal administration of natural betainterferon. J Neurol Neurosurg Psychiatry. 1986;49:1308-12.

19. Yu X, Burgoon M, Green M, Barmina O, Dennison K, Pointon T, et al. Intrathecally synthesized lgG in multiple sclerosis cerebrospinal fluid recognizes identical epitopes over time. J Neuroimmunol. 2011;240-241:129-36.

20. Ragheb S, Li Y, Simon K, VanHaerents S, Galimberti D, De Riz M, et al. Multiple sclerosis: BAFF and CXCL13 in cerebrospinal fluid. Mult Scler. 2011;17:819-29.

21. Brettschneider J, Czerwoniak A, Senel M, Fang L, Kassubek J, Pinkhardt E, et al. The chemokine CXCL13 is a prognostic marker in clinically isolated syndrome (CIS). PLoS One. 2010;5:e11986.

22. Sellebjerg F, Bornsen L, Khademi M, Krakauer M, Olsson T, Frederiksen $J$, et al. Increased cerebrospinal fluid concentrations of the chemokine CXCL13 in active MS. Neurology. 2009;73:2003-10.

23. Khademi M, Kockum I, Andersson ML, lacobaeus E, Brundin L, Sellebjerg F, et al. Cerebrospinal fluid CXCL13 in multiple sclerosis: a suggestive prognostic marker for the disease course. Mult Scler. 2011;17:335-43.

24. Kothur K, Wienholt L, Brilot F, Dale RC. CSF cytokines/chemokines as biomarkers in neuroinflammatory CNS disorders: a systematic review. Cytokine. 2016;77:227-37.

25. Seppi D, Puthenparampil M, Federle L, Ruggero S, Toffanin E, Rinaldi F, et al. Cerebrospinal fluid IL-1 $\beta$ correlates with cortical pathology load in multiple sclerosis at clinical onset. J Neuroimmunol. 2014;270:56-60.

26. Krumbholz M, Theil D, Cepok S, Hemmer B, Kivisäkk P, Ransohoff RM, et al. Chemokines in multiple sclerosis: CXCL12 and CXCL13 up-regulation is differentially linked to CNS immune cell recruitment. Brain. 2006;129:200-11. 